\title{
Mécanique de l'érosion des vases
}

\section{The mechanics of mud erosion}

\author{
Claire Périgaud
}

Institut de physique du globe de Paris

Laboratoire de physique et chimie de l'hydrosphère

\section{Introduction}

L'érosion des vases est une question depuis longtemps abordée, par exemple pour les aménagements de ports dans les estuaires. Elle est analysée dans le cadre des études de faisabilité des projets de dépôts de déchets nucléaires dans les fonds océaniques. L'étude des couches sédimentaires sous-marines permet également de reconstituer l'histoire des courants océaniques profonds dans la mesure où les seuils d'érosion et de dépôt sont connus.

Dans cette étude, les différents aspects mécaniques de l'érosion des sédiments cohésifs sont abordés principalement à partir des études expérimentales de la littérature. La première partie présente les différents modèles rhéologiques qui permettent de comprendre le comportement d'une vase soumise à une action mécanique donnée.

La deuxième partie permet de comprendre comment le courant agit sur le fond.

Enfin, la troisième partie décrit les processus d'érosion : ceux-ci sont différents selon que la vase est concentrée ou non; une interprétation de ces mécanismes est alors proposée.

\section{Rhéologie des vases}

Les vases considérées ici sont des sols fins saturés en eau, c'est-à-dire un mélange d'eau et de grains de taille inférieure à 100 microns. Ce sont des sédiments cohésifs par opposition aux sédiments grossiers dont le comportement ne dépend que des frottements mécaniques entre les grains; les vases ont une rhéologie beaucoup plus complexe que celle des sables. Dans ce paragraphe, quelques modèles rhéologiques des vases sont décrits en premier lieu; les facteurs influençant ce comportement sont ensuite mentionnés; enfin, une synthèse des résultats expérimentaux publiés sur la rhéologie des vases permet de retenir deux types de comportement différents.

\subsection{Modèles de comportement rhéologique des vases}

De nombreux paramètres entrent en jeu dans le comportement d'une vase en réponse à une action mécanique donnée. Retenons pour l'instant que le paramètre clé de ce comportement est la concentration $T_{s}(\mathrm{~g} / \mathrm{l})$ qui mesure le poids de sédiments secs contenus dans un volume de vase.

Très schématiquement, on peut distinguer en fonction de la valeur de la concentration, deux types de comportement : un comportement de type fluide où la vase est considérée comme un milieu monophasique, et un comportement de type solide déformable où l'eau interstitielle et l'ensemble des grains forment deux phases distinctes.

\section{Faibles concentrations en grains}

Pour les faibles concentrations, l'étude du comportement des vases, alors de type fluide, se fait au viscosimètre ou appareils dérivés, tels que le viscosimètre Brookfield (Fig. 1a) et le viscosimètre à palette (Fig. 1b). Le principe est de mesurer le couple de torsion exercé par la vase en réponse à une vitesse de rotation donnée. Les différents types de courbes obtenues peuvent être représentés par les modèles suivants :

Modèle visqueux linéaire (Fig. $2 \mathrm{a}$ ) : Le couple de torsion est proportionnel à la vitesse de rotation, c'est-à-dire que la contrainte de cisaillement. $\tau$ dans la vase est proportionnelle à la vitesse de cisaillement $d u / d y$ soit $\tau=\mu$ $d u / d y$ où $\mu$ est le coefficient de viscosité dynamique $\left(\mathrm{kg} \mathrm{m}^{-1} \mathrm{~s}^{-1}\right)$.

LA HOUILLE BLANCHE/N ${ }^{\circ} 7 / 8-1983$ 

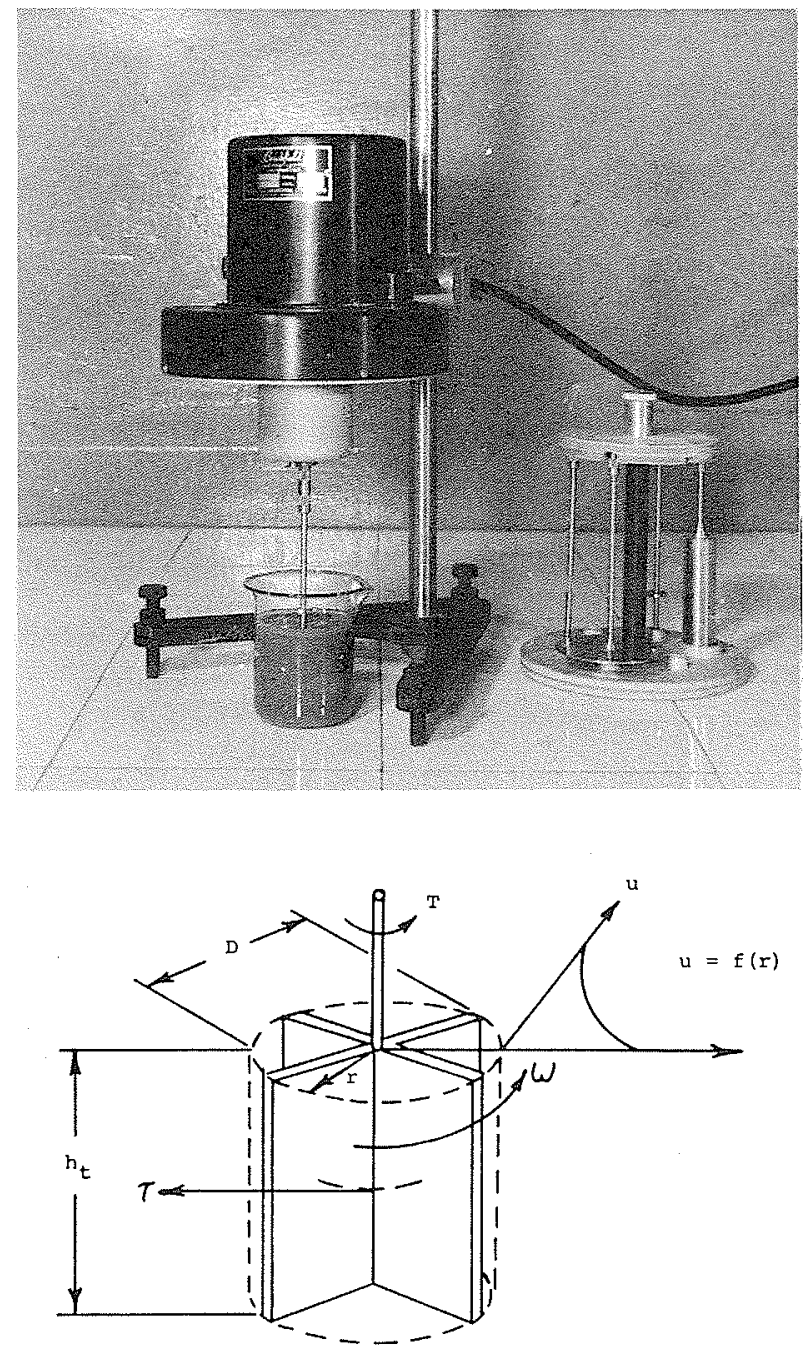

Figure 1. - Deux types de viscosimètres différents pour étudier la résistance au cisaillement de vases peu (Fig. 1a) ou très (Fig. 1b) concentrées.

Figure 1a : Viscosimètre Brookfield (Migniot, 1970)

Figure 1b: Viscosimètre à palette (Gularte, 1978)

Pour un problème à trois dimensions, le tenseur de contrainte $\underline{\sigma}$ est relié à la pression $p$ et au tenseur de vitesse de déformation $d$ par la loi :

$$
\underline{\underline{\sigma}}=-p \underline{\underline{I}}+2 \mu \underline{\underline{d}}
$$

Modèle pseudoplastique (Fig. 2b) : La viscosité apparente, définie comme tangente à la courbe $(\tau, d u / d y)$, diminue lorsque la vitesse de cisaillement augmente. Ce comportement pseudoplastique est souvent illustré par une loi en puissance, c'est-à-dire :

$$
\tau=\alpha(d u / d y)^{m}
$$

$\alpha$ est un coefficient de dimension $M L^{-1} T^{m-2} ; m$ est un exposant sans dimension compris entre 0 et 1 .

Pour un problème à trois dimensions, cette loi correspond au modèle de Norton-Hoff (Hoff 1954):

$$
\underline{\underline{g}}=-p \underline{\underline{I}}+\left(I_{2}\right)^{(m-1) / 2} \underline{\underline{d}}
$$

où $I_{2}$ est le second invariant du tenseur vitesse de déformation $\left(I_{2}=(1 / 2) \times \operatorname{tr}\left(\underline{\underline{d}}^{2}\right)\right)$.

Modèle viscoplastique (Fig. 2c) : Une contrainte minimun est nécessaire avant de mettre en mouvement le fluide : au-delà de ce seuil de plasticité, le comportement de la vase peut être linéaire (modèle de Bingham); il est alors défini par la viscosité dynamique $\mu_{B}$ :

$$
\tau=\tau_{B}+\mu_{B} d u / d y
$$

Pour un problème à trois dimensions, la loi ci-dessus s'écrit (Oldroyd 1947):

$$
\begin{aligned}
& \text { si } \quad J_{2}<\tau_{B}^{2} \underset{m}{d}=0 \\
& \text { si } \quad J_{2} \geqslant \tau_{B}^{2} \underline{\sigma}=-p \underline{I}+2 \mu^{*} \underset{\alpha}{d}
\end{aligned}
$$

où $J_{2}$ est le second invariant du déviateur des contraintes $s: J_{2}=(1 / 2) \times \operatorname{tr}\left(\underline{s}^{2}\right)$ avec $\underline{s}=\underline{\sigma}+p \underline{I} ; \mu^{*}$ est la viscosité newtonienne équivalente : $\mu^{*}=\mu_{B}+\tau_{B} /\left(2 \sqrt{I_{2}}\right)$.

Le modèle de Bingham est le modèle viscoplastique le plus simple; le modèle de Casson définit un raccord parabolique avec le seuil de plasticité $\tau=\tau_{0}+\sqrt{k d u / d y}$

Notons enfin que les modèles décrits ci-dessus sont très simples : le comportement réel de la vase est souvent thixotropique, c'est-à-dire que pour une même vitesse de cisaillement, la viscosité apparente diminue avec le temps et reprend sa valeur initiale après un repos suffisamment long (Fig. 2d) (Schowalter 1978). Il y a fausse thixotropie lorsque ces modifications ne sont pas réversibles.

a
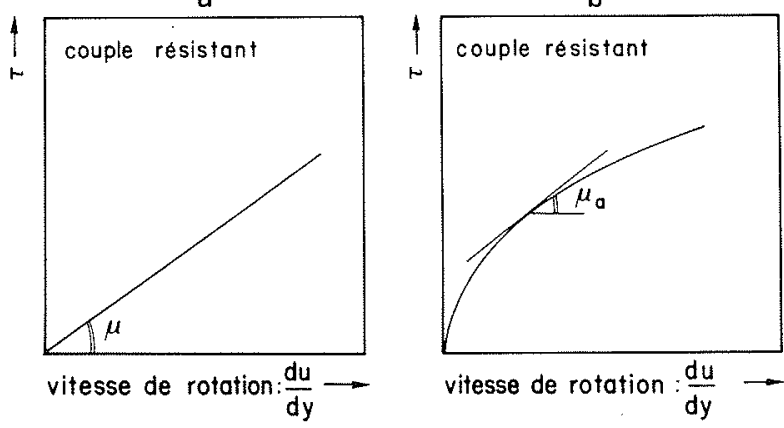

d
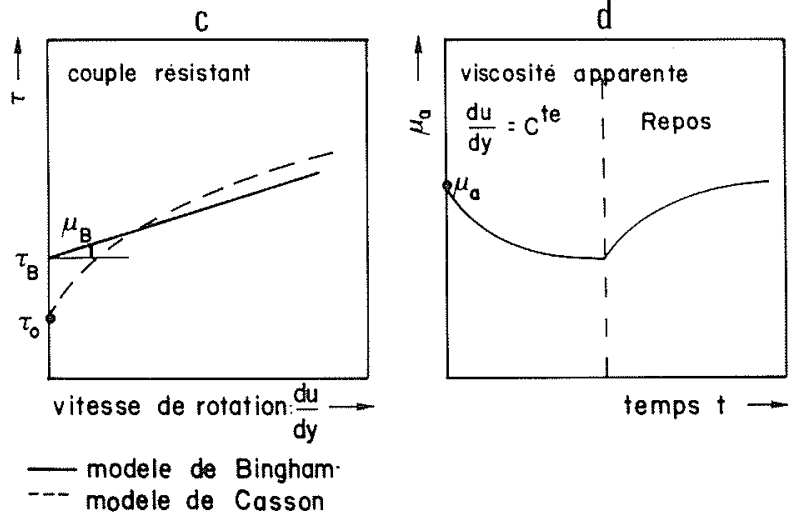

le de Bingham

Figure 2. - Schématisation de modèles de comportement simples applicables aux vases peu concentrées. Courbes obtenues pour des essais au viscosimètre.

Figure 2a : fluide visqueux newtonien

Figure $2 b$ : fluide pseudoplastique

Figure $2 c$ : fluide viscoplastique

Figure 2d : fluide thixotropique. 


\section{Fortes concentrations en grains}

Pour les fortes concentrations de grains, l'étude du comportement des vases, alors de type solide, se fait avec un scissomètre par exemple. Le principe est de mesurer le couple de torsion $\tau$ exercé par la vase en réponse à une déformation angulaire donnée $\epsilon$. Les courbes obtenues ont l'allure représentée sur la figure $3 a$. La première partie de la courbe est linéaire et correspond à un comportement élastique dont le module de cisaillement $G$ est donné par la pente de courbe $(\tau, \epsilon)$. Le maximum de contrainte, appelée cohésion non drainée $C_{u}$, correspond à la rupture. Coulomb a défini un critère reliant la contrainte normale $\sigma$ et la contrainte tangentielle $\tau$ s'exerçant sur une facette lorsque le seuil de rupture $y$ est atteint (Fig. 3b) :

$$
\tau=C-\sigma \operatorname{tg} \dot{\varphi}
$$

$C$ est la cohésion effective (cohésion obtenue dans un essai consolidé drainé correspondant à un comportement à long terme); $\phi$ est l'angle de frottement interne ; (dans tout ce papier, les contraintes sont comptées positive. ment en traction).

Le comportement des vases concentrées avant la rupture est cependant très mal connu. Le principe de Terzaghi (Terzaghi 1943) consiste à décomposer le tenseur des contraintes totales $\sigma$ en un tenseur de contraintes effectives $\underline{\sigma}^{*}$ s'exerçant sur le squelette solide déformable constitué par les grains et en pression de l'eau interstitielle $p: \underline{\underline{\sigma}}=\underline{\underline{\sigma}}^{*}-p \underline{\underline{\underline{I}}}$. Lorsque le squelette répond linéairement à une déformation, le comporte-

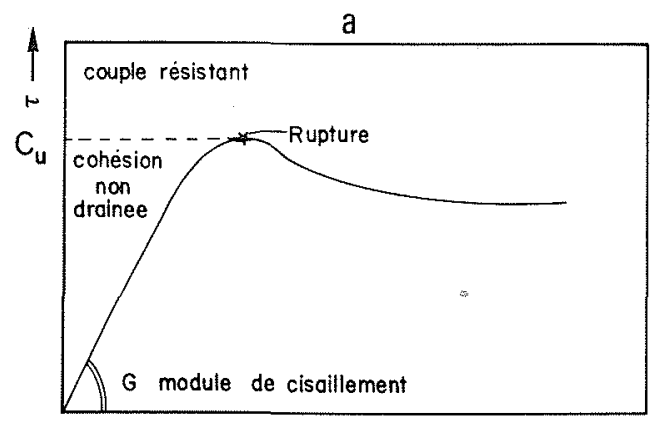

angle de rotation (deformation $\epsilon_{x^{\prime} y}$ ) $\longrightarrow$

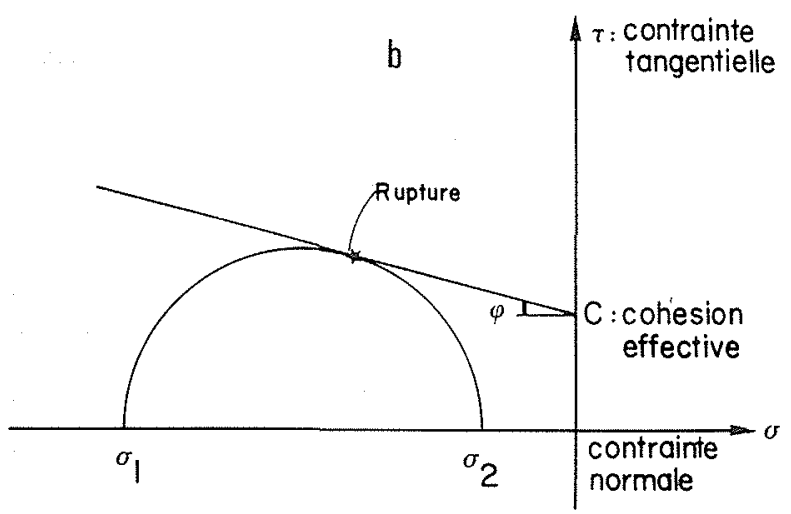

Figure 3. - Schématisation de modèles de comportement simples applicables aux vases concentrées.

Figure 3a : Comportement de type solide déformable élastiquement avant rupture. Courbe obtenue pour des essais au scissomètre.

Figure 3b: Cercle de Mohr et critère de rupture de Coulomb. ment peut être modélisé par les lois de l'élasticité $\underline{\underline{\sigma}}^{*}=2 G\{\underline{\underline{\epsilon}}+(\nu / 1-2 \nu) \operatorname{tr} \epsilon \times I\}$ où $\underline{\underline{e}}$ est le tenseur de déformation du squelette solide, $G$ est le module de cisaillement et $\nu$ le coefficient de Poisson. Enfin, l'écoulement de l'eau à travers le squelette est défini par la loi de Darcy (Schlosser, 1978) : les vitesses relatives entre l'eau et les grains sont proportionnelles au gradient de pression qui les induit en régime permanent; la constante de proportionnalité est le coefficient de perméabilité intrinsèque $\left(\mathrm{ms}^{-1}\right)$ (voir aussi Gilbert 1983).

\subsection{Facteurs influençant le comportement rhéologique des vases}

Outre la concentration $T_{s}$ qui joue un rôle déterminant, les facteurs influençant le comportement rhéologique d'une vase sont multiples.

La nature minéralogique de la vase (Migniot 1968, 1977 ) et le pourcentage de matière organique, en particulier la bioturbation (Richards et Parks 1976) entrent en jeu.

Les vases sont généralement constituées de plusieurs matériaux dont la granulométrie va en décroissant depuis les sables jusqu'aux colloïdes $\vdots$ à concentration égale, la cohésion(1) augmente avec la petitesse des grains (Migniot 1968).

La cohésion* est le résultat du frottement entre les grains et de forces de répulsion ou d'attraction entre particules d'eau et de vase : les paramètres physicochimiques tels que la présence d'ions floculants, un $\mathrm{pH}$ basique et la salinité renforcent la cohésion* tandis qu'une élévation de température l'affaiblit (Migniot 1968 ; Partheniades 1965; Gularte 1978; O'Neil 1979).

L'histoire de la vase, en particulier, son mode de dépôt, modifie considérablement ses propriétés mécaniques : ceci est dû à des effets de fausse thixotropie.

\subsection{Synthèse des résultats sur la rhéologie}

Les résultats qui suivent, utilisent les études de plusieurs auteurs. Une liste en est donnée dans le tableau I.

\begin{tabular}{|c|c|}
\hline Tableau 1. - & $\begin{array}{l}\text { Liste des études consultées sur la } \\
\text { rhéologie des vases }\end{array}$ \\
\hline Chavarria (1980) & $\begin{array}{l}\text { : Rhéologie des boues biologiques très peu } \\
\text { : concentrées; comportement non linéaire }\end{array}$ \\
\hline $\begin{array}{l}\text { Migniot (1968) } \\
\text { Valembois (1975) }\end{array}$ & $\begin{array}{l}\text { Seuil de Bingham et viscosité apparente } \\
: \text { - flocculation - tassement - teneur en } \\
: \text { sable }\end{array}$ \\
\hline $\begin{array}{l}\text { Chong (1971) } \\
\text { Thomas (1963) } \\
\text { Jeffrey (1976) }\end{array}$ & $\begin{array}{l}\text { Seuil de Bingham et viscosité apparente } \\
\text { : en fonction de la concentration pour des } \\
\text { : milieux diphasiques }\end{array}$ \\
\hline $\begin{array}{l}\text { Biarez (1971), } \\
\text { Gérard (1978), } \\
\text { Silva (1976), } \\
\text { Richards (1976) }\end{array}$ & $\begin{array}{l}\text { Synthèse des connaissances sur les pro- } \\
\text { : priétés des sédiments en mer profonde. } \\
\text { : Propriétés des sédiments en mer pro- } \\
\text { fonde. } \\
\text { : Données géotechniques et mécaniques. }\end{array}$ \\
\hline
\end{tabular}

(1) dans ce paragraphe, nous utilisons le mot cohésion* sans préciser à quel paramètre il est fait référence (viscosité, newtonienne ou apparente, seuil de Bingham, cohésion effective...). 
Etant donnée la multiplicité des facteurs entrant en jeu et la diversité des méthodes de mesures, les principes qui en sont retirés et décrits ci-dessous sont délibérément simplifiés pour permettre une approche de modélisation des mécanismes d'érosion (Périgaud 1983).

Le facteur déterminant du comportement rhéologique des vases est la concentration en grains $T_{s}$.

\section{Faibles concentrations en grains}

Pour des vases de faible concentration en grains telles que les crèmes de vase en estuaire ou les dépôts superficiels marins par exemple, le modèle le plus fréquemment retenu est celui de Bingham. Les valeurs trouvées par différents auteurs sur le seuil de Bingham $\tau_{B}$ et sur la viscosité $\mu_{B}$ en fonction de la concentration $T_{s}$ sont rassemblées dans les tableaux II et III ; en général, les auteurs proposent une relation puissance entre $\tau_{B}$ et $T_{s}: \tau_{B}=k T_{s}^{p}$ avec $p$ compris entre 3 et 4 .

Cependant, les courbes expérimentales ne présentent jamais l'aspect parfait du modele de Bingham. En particulier, la mise en mouvement de la vase à partir d'un seuil de contrainte minimum est délicate à détecter et dépend du type d'appareil utilisé (Fig. 4). Dans ces conditions, il est plus simple de retenir pour une approche mécanique de l'érosion des vases un modèle de fluide visqueux non linéaire (loi puissance $\tau=\alpha(d u / d y)^{m}$ ). A partir de ce modèle, Chavarria (1980) a déterminé expérimentalement les variations de l'exposant $m$ et du coefficient multiplicateur en fonction de la concentra-

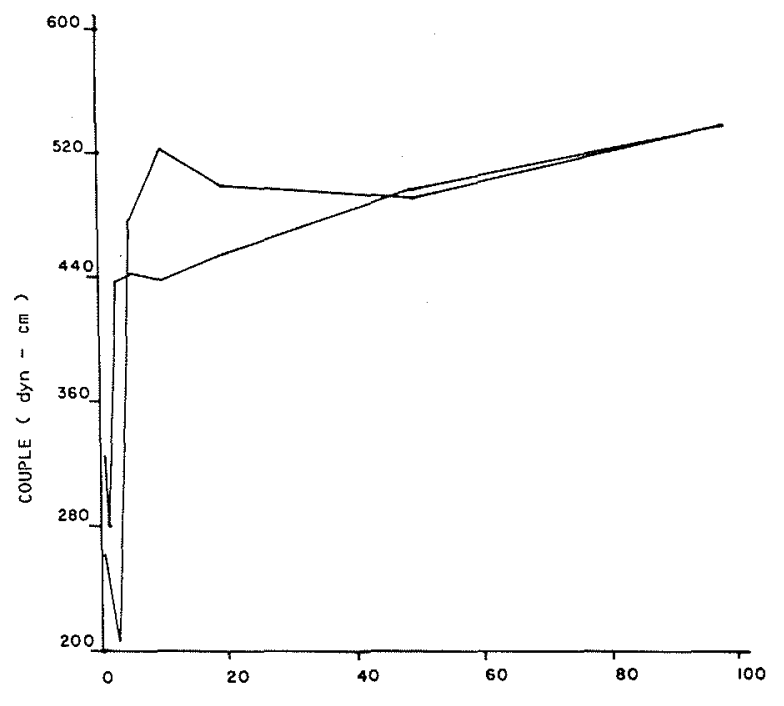

VITESSE DE ROTATION (Nombre de tours par minute)

Figure 4. - Exemple de courbe expérimentale obtenue au viscosimètre à palette (Clanton, 1979).

tion $T_{s}$ pour des vases très peu concentrées. Les valeurs moyennées sur les différents essais, recalculées avec notre définition de $\alpha$ et de $m$ sont (exprimées en unités S.I.) :

$$
m=1 /(0,058 \times C-1,042) \text { soit } 0,26<m<0,36
$$

Tableau II. - Valeurs du seuil de Bingham $\tau_{B}(\mathrm{~Pa})$ en fonction de la concentration $T_{s}(\mathrm{~g} / \mathrm{l})$ pour différents auteurs

\begin{tabular}{|c|c|c|c|c|c|c|c|c|c|c|c|c|c|c|}
\hline Auteurs & 100 & 150 & 200 & 250 & 300 & 350 & 400 & 450 & 500 & 600 & 700 & 800 & 900 & 1000 \\
\hline Migniot $\begin{array}{l}\text { Mahury } \\
\text { Provins }\end{array}$ & 0,01 & 0,08 & $\begin{array}{l}0,25 \\
0,02\end{array}$ & $\begin{array}{l}0,80 \\
0,07\end{array}$ & $\begin{array}{l}1,50 \\
0,11\end{array}$ & $\begin{array}{l}5,00 \\
0,30\end{array}$ & $\begin{array}{l}8,00 \\
0,70\end{array}$ & $\begin{array}{c}12,0 \\
1,0\end{array}$ & $\begin{array}{l}20 \\
1,8\end{array}$ & $\begin{array}{r}50 \\
5\end{array}$ & $\begin{array}{r}100 \\
10\end{array}$ & 20 & & \\
\hline Gironde & & & 0,16 & 0,25 & 0,4 & 0,7 & 1,4 & 2,0 & 1,7 & & . & & & \\
\hline Gularte & 2,0 & 10 & & 20 & & & & & 35 & 50 & 70 & & 130 & 200 \\
\hline Clanton & 0,3 & & & & 5,0 & & & & 40 & & 200 & & 2000 & \\
\hline Valembois & & & 0,06 & & & 0,33 & & 1,25 & 2,27 & & & & & \\
\hline
\end{tabular}

Tableau III. - Valeurs de la viscosité apparente (Poise $=10^{-1} \mathrm{SI}$ ) en fonction de la concentration $T_{s}(\mathrm{~g} / \mathrm{l})$ pour différents auteurs

\begin{tabular}{|c|c|c|c|c|c|c|c|c|c|c|c|c|c|c|c|}
\hline \multicolumn{1}{|c|}{$T_{s}$} & 50 & 100 & 150 & 200 & 250 & 300 & 350 & 400 & 450 & 500 & 600 & 700 & 800 & 1000 & \\
\hline $\begin{array}{r}\text { Muteurs } \\
\text { Migniot Mahury } \\
\begin{array}{r}\text { Provins } \\
\text { Gironde }\end{array}\end{array}$ & 0,02 & 0,03 & 0,08 & 0,15 & 0,80 & $\begin{array}{c}2,00 \\
0,06\end{array}$ & & $\begin{array}{c}8,00 \\
0,10\end{array}$ & & $\begin{array}{c}20 \\
0,4\end{array}$ & $\begin{array}{c}80 \\
0,8\end{array}$ & & 1 & & \\
\hline Gularte & & 0,3 & & & & 0,4 & & & & & & 40 & & 700 & \\
\hline Clanton & 0,01 & 0,01 & 0,01 & 0,2 & & 0,4 & & 0,5 & & 0,8 & 1,5 & 5 & & & \\
\hline
\end{tabular}

Ces valeurs sont recalculées à partir des courbes expérimentales qui ne dépendent pas toujours de $T_{S}$ mais parfois de la teneur en eau ou du poids efíectif de la vase. Lorsque le $\mathrm{pH}$, la température et la salinité varient, nous avons retenu les valeurs pour $\mathrm{pH}=6$, température $=20^{\circ} \mathrm{C}, S=0 \%$. 
et :

$$
\alpha=10^{a \circ(1-m)}-4
$$

$a_{\mathrm{o}}=0,494+2,8 \log C$ soit $6 \times 10^{-3}<\alpha<0,65$

$C$ est le pourcentage de matière en suspension

$$
\left(C=100 \times T_{s} / 1000\right)
$$

$C$ est compris entre 0,5 et 5 dans les expériences de Chavarria (1980).

\section{Fortes concentrations en grains}

Pour les vases concentrées telles qu'on les rencontre au fond des océans en dessous des premiers centimètres de sédiments par exemple, les grandeurs mécaniques mesurées in situ (Silva et Hollister 1979) ou en laboratoire sur échantillon (Silva et al. 1975; Gérard 1978) sont : les teneurs en eau $w$, les limites de liquidité $w_{L}$ et de plasticité $w_{p}$, la cohésion non drainée $C_{u}$ et l'angle de frottement interne $\phi$, le rapport de surconsolidation et l'indice de compressibilité $C_{C}$. On trouvera dans Biarez (1977) et dans Richards et Parks (1976) des valeurs moyennes, minima et extrêma de ces paramètres dans les différents océans.

Retenons que en moyenne, ce sont des matériaux très fins, à teneur en eau et résistance au cisaillement relativement élevées $\left(w \sim 150 \%, C_{u} \sim 10 k P a, \phi \sim 30^{\circ}\right)$. En réalité, les sédiments sont très stratifiés et leurs caractéristiques mécaniques dépendent donc également de la profondeur. L'indice de compressibilité donne une idée de la déformabilité de la vase $\left(C_{C}=1\right.$ en moyenne) mais les données sur la perméabilité et l'élasticité du matériau sont beaucoup plus rares; en fait, le choix d'un modèle élastique pour le comportement du squelette solide est irréaliste ; c'est cependant le choix fait dans les approches théoriques de mécanismes tels que la rupture de couches sédimentaires sous l'action de la houle (Yamamoto 1977 ; Madsen 1978; Mei 1980 ; Périgaud, 1983). Des ordres de grandeur raisonnables pour le coefficient de perméabilité intrinsèque $K$, le module de cisaillement $G$ et le module de Poisson $\nu$ sont :

$$
K \sim 10^{-4} \mathrm{~m} / \mathrm{s} ; G \sim 10^{7} \mathrm{~Pa} ; \nu \sim 0,3
$$

\section{Action du courant sur le fond}

Le paragraphe précédent tentait de répondre à la question : quel est le comportement de la vase sous une action mécanique donnée? Pour savoir comment les vases sont érodées par un courant horizontal $V$ donné, il faut connaître aussi les contraintes qu'exerce un tel courant sur le fond. Après quelques rappels sur la couche limite d'un écoulement turbulent sur fond plat, sont rappelées ici les méthodes de détermination des contraintes exercées sur le fond. En fait, ce point fait l'objet de recherches actuelles, en particulier sur les bouffées turbulentes qui heurtent le fond périodiquement : quelques éléments en sont décrits à la fin de ce paragraphe.

\subsection{Couche limite turbulente sur plaque plane}

Que ce soit dans les océans, les estuaires ou les rivières, un courant apte à éroder les sédiments est toujours turbulent : il peut être modélisé par un courant $V$ moyen horizontal permanent et des fluctuations de vitesse tridimensionnelles $u^{\prime}, \vec{v}^{\prime}, \dot{w}^{\prime}$ ainsi que de pression $p^{\prime}$. La présence de la paroi se fait sentir sur une hauteur $\delta$, épaisseur de la couche limite, dans laquelle la vitesse $V$ est cisaillée. Sur le plan théorique, cette couche limite se décompose en plusieurs sous-couches qui correspondent aux zones de prépondérance des différents termes en jeu (inertie - gradient de pression - frottement turbulent et frottement visqueux moléculaire). La structure de cette couche limite, résumée dans le tableau 4, est détaillée dans Schlichting (1979) et Darrozès et François (1982). Dans cette hypothèse, les contraintes exercées sur le fond par cet écoulement sont des contraintes de cisaillement $\tau_{0}$ :

$$
\tau_{0}=\mu d V / d z(0)=\rho u_{*}^{2}
$$

$\rho$ et $u$ sont la masse volumique et la viscosité dynamique de l'eau; $z$ est la hauteur au-dessus du fond; $u_{*}$ est appelée vitesse de frottement.

\subsection{Frottement sur la paroi}

Il y a trois méthodes possibles pour déterminer la contrainte de frottement $\tau_{0}$.

Utilisation du coefficient local de frottement $C_{f}$ : Ce coefficient, $C_{f}=2 \tau_{0} / \rho V^{2}$ par définition, est exprimé à l'aide de différentes formules hydrodynamiques. Il ne faut pas s'étonner de la multiplicité des formules proposées pour ce paramètre :

Le principe des méthodes intégrales approchées (Darrozès-François, 1982) consiste à relier la contrainte de frottement $\tau_{0}$ à l'intégration du profil de vitesse à travers toute la couche limite; les différents profils choisis étant approchés par rapport à la réalité, les formules obtenues sont approximativement équivalentes parce que les écarts sont lissés par l'intégration.

Schlichting (1979) propose pour la couche limite d'épaisseur $\delta$ :

$$
C_{f}=0,0074 / R e^{(1 / 5)}
$$

où $R e$ est le nombre de Reynolds $R e=\rho V \delta / \mu$

Utilisation du profil de vitesse moyenne : Dans la sous-couche logarithmique (tableau IV), la vitesse moyenne est théoriquement connue (Schlichting 1979): $V(z)=\left(u_{*} / K\right) \log \left(z u_{*} / \nu\right)$ où $K$ est la constante de Karman (voisin de 0,4$)$. La pente du profil tracé dans un diagramme semi-logarithmique donne la valeur de $u_{*}$, donc de $\tau_{0}$. Retenons que dans une couche limite turbulente, $u_{*}$ est de l'ordre de $V / 30$.

En fait, la formule du profil de vitesse ci-dessus est modifiée lorsque le fond n'est pas lisse :

$$
V(z)=\left(u_{*} / K\right) \log (z / h)+C
$$

où $h$ et $C$ sont des fonctions de la hauteur des aspérités du fond.

Utilisation des fluctuations de vitesses : La mesure la plus directe de la contrainte se fait au film chaud ou au laser en calculant le produit des fluctuations de vitesses $u^{\prime}$ et $w^{\prime}$ dans la couche à flux constant (tableau IX); en effet, dans cette couche, la contrainte $\tau$ est indépendante de la distance à la paroi $z$ et vaut :

$$
\tau_{(z)}=\tau_{0}=d V / d z(z)-\rho \overline{u^{\prime} w^{\prime}}(z)
$$




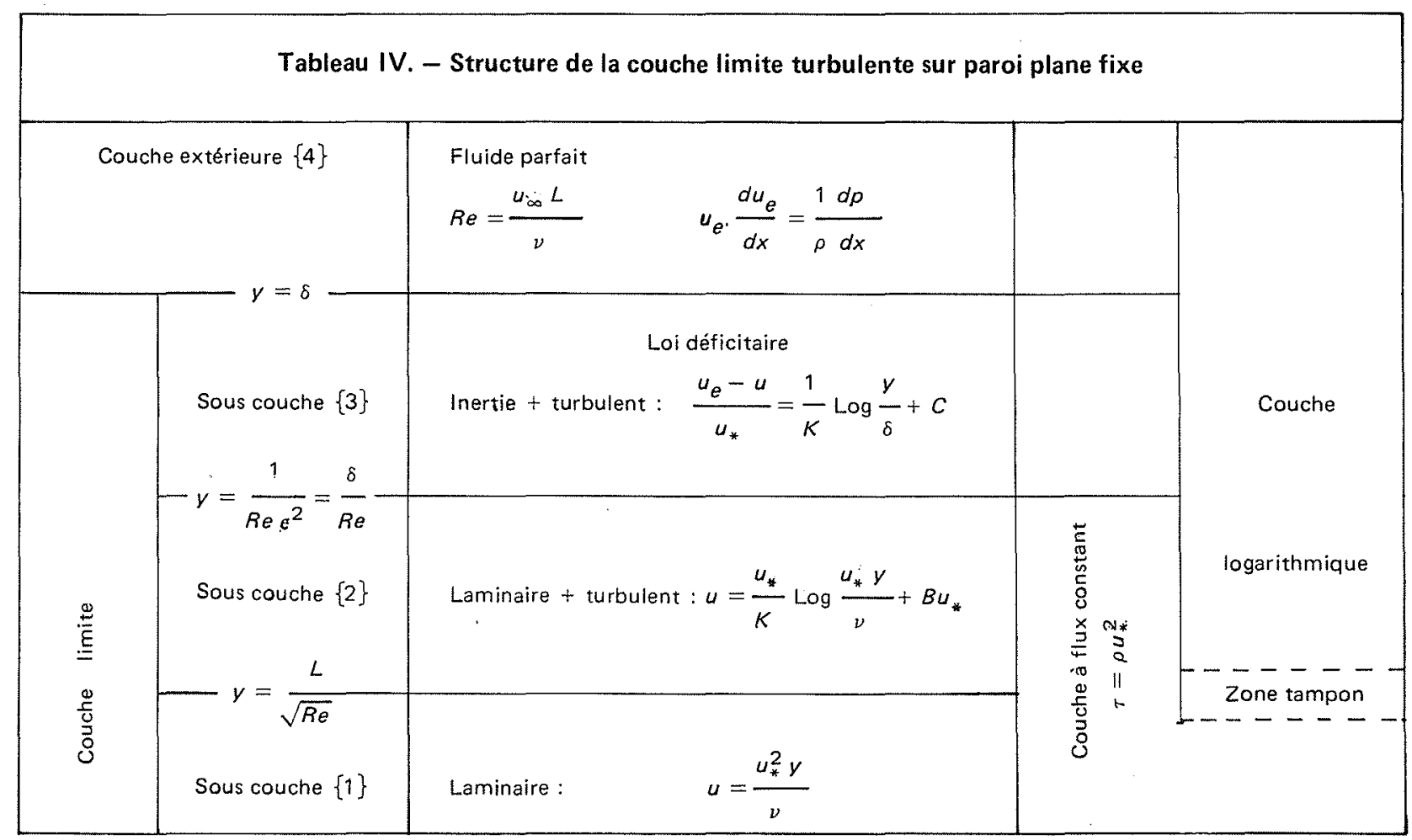

Le calcul direct de ce produit est cependant difficile (il nécessite la mesure simultanée de $u^{\prime}$ ef $v^{\prime}$, đonc deux lasers ou un film chaud croisé); il est le plus souvent erroné, à cause des perturbations de fréquence induites par de nombreux facteurs extérieurs à l'écoulement. Il est alors beaucoup plus raisonnable de déterminer la vitesse de frottement $u_{*}$ à partir du taux de dissipation d'énergie cinétique turbulente; en effet ce taux est caractéristique du stade ultime de transfert de l'énergie vers les petites échelles et il est indépendant des mécanismes de production de la turbulence déterminant la structure macroscopique de celle-ci. Ce taux, qui vaut $u_{*}^{3} /(K z)$ au-delà de la sous-couche laminaire, est déterminé à l'aide du spectre en nombre d'onde des fluctuations de vitesse (Monin et Yaglom 1971).

\subsection{Les bouffées turbulentes}

La description théorique précédente est simplifiée par rapport à la réalité : il n'existe pas de sous-couche laminaire (tableau IV); celle-ci est périodiquement détruite par la présence de bouffées turbulentes ("bursts" en anglais) qui génèrent des fluctuations de vitesse, de pression et de cisaillement jusqu'au fond.

Il se trouve que la structure de ces bouffées turbulentes n'est pas aléatoire mais obéit à un cycle dans le temps et l'espace bien déterminé et cohérent. On appelle "bouffée" une séquence d'écoulement depuis la naissance de celle-ci jusqu'à sa disparition. Il semblerait que ces "bouffées" naissent de l'interaction de tourbillons de grande échelle de l'écoulement extérieur à la couche limite et des tourbillons de plus petite échelle à l'intérieur de celle-ci. Doligaski et al. (1979) étudient numériquement la convection d'un tourbillon dans un écoulement cisaillé de couche limite : la présence d'un tel tour- billon induit la naissance d'un tourbillon "fils" dans la sous-couche laminaire. Ce tourbillon "fils" grandit à son tour et dépasse la hauteur caractéristique de la souscouche laminaire pour "éclater" dans la sous-couche turbulente.

Cantwell (1981) résume les différentes étapes expérimentales qui ont permis de dégager les résultats suivants :

- une "bouffée" prend naissance à une distance $z$ audessus du fond de l'ordre de 5 à $15 \nu / u_{*}$ (cette zone correspond à la zone tampon du tableau 4); notons qu'à cette hauteur, la vitesse est telle qu'elle correspond à l'apparition de la première instabilité de l'écoulement cisaille : le nombre de Reynolds critique de stabilité de l'écoulement de couche limite vaut $R_{C}=420$ (Darrozès et François, 1982, p. 336); dans la sous-couche laminaire, la vitesse a pour expression : $u(z)=z\left(u_{*}^{2} / v\right)$; or le nombre de Reynolds $R e=z\left(z u_{*}^{2} / v\right) / \nu$ vaut 420 à la hauteur $z=20 v / u_{*}$;

- suit la pénétration du fluide à quantité de mouvement élevée dans la couche inférieure ; cette phase est appelée "INRUSH" (Fig. 5a) et correspond à la naissance d'un "éclair" à faible vitesse (low-spead streak);

- la paroi est alors balayée dans le sens de l'écoulement moyen. Cette phase est appelée "SWEEP" (Fig. 5a) et correspond à l'allongement de l' "éclair". La contrainte de cisaillement à la paroi est alors maximum et peut atteindre 30 à 100 fois la valeur moyenne $\tau_{0}$ (Willmarth et $\mathrm{Lu}, 1972$ );

- le fluide de quantité de mouvement alors diminuée est réjecté dans les couches supérieures : cette phase est appelée "EJECTION" (Fig. 5b) et correspond à la déformation tridimensionnelle de l' "éclair";

- après quelques oscillations, la bouffée "éclate"; cette phase est appelée "BREAK-UP" (Fig. 5b). 


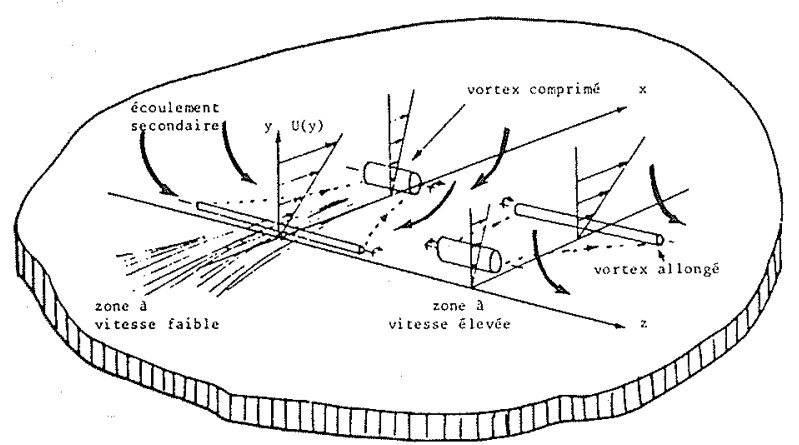

a

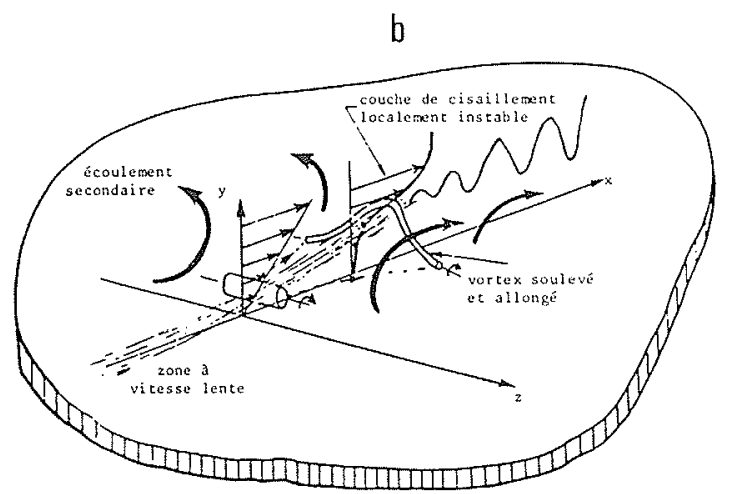

Figure 5. - Cycle d'une bouffée turbulente (Kline et al., 1967).

Figure 5a : Naissance de l'éclair. Phases de pénétration ("inrush") puis de balayage ("sweep")

Figure 5b: Disparition de l'éclair. Phases d'éjection ("ejection") et d'éclatement ("break-up").

Bien que le phénomène soit assez complexe, on peut retenir que l'ensemble des séquences, depuis l" INRUSH" jusqu'au "BREAK-UP", est un phénomène cyclique dont la période $T_{b}$ est évaluée à environ $5 \delta / V$ et la longueur d'onde dans le sens du courant $\lambda_{b}$ vaut entre 700 et $1500 \nu / u_{*}$.

Retenons enfin que la conséquence de l'existence de ces bouffées est une contribution majeure, bien qu'intermittente, à la tension de cisaillement sur le fond.

\section{Erosion des vases}

Les études expérimentales sur l'érosion des vases sont nombreuses. Les résultats obtenus ne sont pas toujours comparables vu la multiplicité des paramètres entrant en jeu. Le tableau $\mathrm{V}$ donne la liste des études consultées avec leur spécificité.

Ces études montrent qu'il existe une vitesse critique du courant à partir de laquelle les sédiments sont érodés. Nous montrons d'abord que l'ordre de grandeur de cette vitesse est incompatible avec les deux mécanismes suivants : arrachage de particules individuelles et entrainement de vase de type fluide de Bingham. De plus, les observations montrent que l'érosion est un phénomène intermittent et qu'elle relève de deux mécanismes différents suivant que la vase est concentrée ou non. Nous proposerons alors une modélisation de ces deux mécanismes.

\section{Tableau V. - Liste des études consultées concernant l'érodibilité des sédiments cohésifs \\ Migniot (1968, : Entraînement par courant, houle ou $\begin{array}{ll}1977) & \text { : vent. Vitesse de frottement critique en } \\ \text { Valembois (1975) : fonction du seuil de Bingham. Taux }\end{array}$ : d'érosion. \\ Lambermont : Taux d'érosion et seuil critique en fonc- (1977) : tion du mode de préparation du lit : Parchure (1980) : dépôt en présence d'un courant de Parsons (1981) : vitesse donnée. \\ Dunn (1959) : Détermination visuelle du seuil critique d'érosion pour une vase remaniée sou- : mise à l'action d'un jet. \\ Sutherland (1966): Simulation de l'entraînement turbulent : par un jet traversier. \\ Moore et Mash : Erosion d'un échantillon cylindrique de (1962) : vase placé dans un cylindre tournant. : Mesure du taux d'érosion à partir de la : perte de poids de l'échantillon. Etude de : I'influence de la salinité différentielle : entre l'eau intersticielle et l'eau du cou- : rant. \\ Krone (1962) : Etude du taux d'érosion en fonction de : la contrainte de cisaillement exercée par : un courant.}

\section{Etudes des paramètres physico-chimiques}

\begin{tabular}{|c|c|}
\hline $\begin{array}{l}\text { Partheniades } \\
\text { (1965) } \\
\text { Mehta (1979) }\end{array}$ & $\begin{array}{l}\text { Taux d'érosion en fonction de la concen- } \\
\text { : tration de la vase et de la résistance au } \\
\text { : cisaillement. }\end{array}$ \\
\hline $\begin{array}{l}\text { Clanton }(1980) \\
\text { Gularte }(1978) \\
\text { Kelly et al. }(1979)\end{array}$ & $\begin{array}{l}: \text { Etude de l'érosion en terme de processus } \\
: \text { en taux "rate process" détermination de } \\
: \text { la vitesse critique et du taux d'érosion en } \\
: \text { fonction de } E \text { et } V_{f} \text {. }\end{array}$ \\
\hline Ariathurai (1978) & $\begin{array}{l}\text { : Taux d'érosion et seuil critique. Etude } \\
\text { : de l'influence de la capacité en échange } \\
\text { : d'ions et du rapport d'absorption du } \\
\text { : sodium. }\end{array}$ \\
\hline $\begin{array}{l}\text { Arulanandan } \\
(1975)\end{array}$ & $\begin{array}{l}\text { : Etude de l'influence de la teneur en eau } \\
\text { : et de la résistance du cisaillement. }\end{array}$ \\
\hline $\begin{array}{l}\text { Raudkivi- } \\
\text { Hutchinson } \\
\text { (1974) }\end{array}$ & $\begin{array}{l}\text { Influence de la salinité, température, } \\
\text { : potentiel zeta, capacité en échange } \\
\text { : d'ions. }\end{array}$ \\
\hline $\begin{array}{l}\text { McCave (1978) } \\
\text { Young, Southard } \\
\text { (1978) }\end{array}$ & $\begin{array}{l}\text { : Etude de l'érosion des vases dans les } \\
\text { : grands fonds marins. }\end{array}$ \\
\hline $\begin{array}{l}\text { Mei (80), } \\
\text { Madsen (78), } \\
\text { Yamamoto (1977) }\end{array}$ & $\begin{array}{l}\text { : Erosion des fonds marins par la houle. } \\
:\end{array}$ \\
\hline Wan (1982) & $\begin{array}{l}\text { : Arrachage de gros blocs de vase dans la } \\
\text { : Rivière Jaune en Chine. }\end{array}$ \\
\hline
\end{tabular}

\subsection{Vitesse critique d'érosion}

Un ordre de grandeur de la vitesse critique d'érosion d'une vase en fonction de sa concentration est donné dans le tableau VI.

De nombreux paramètres entrent en jeu dans les processus d'érosion des vases.

Outre ceux que nous avons déjà cités comme intervenant au niveau de la rhéologie des vases, la différence en température, $p H$, salinité ou capacité en échange d'ions entre l'eau du courant et l'eau interstitielle de la vase peut également jouer un rôle (Arulanandan et al. 1975). 
Tableau VI. - Vitesse critique d'érosion d'une vase (Migniot, 1968. Vase du Mahury)

\begin{tabular}{|l|c|c|c|c|c|}
\hline$T_{s}(\mathrm{~g} /)$ & 100 & 200 & 300 & 400 & 500 \\
\hline$u_{{ }(\mathrm{cm} / \mathrm{s})}$ & 0,7 & 1,5 & 2,2 & 3,3 & 5,4 \\
$V_{c}(\mathrm{~cm} / \mathrm{s})$ & 20 & 45 & 65 & 100 & 150 \\
\hline
\end{tabular}

Une approche possible pour étudier cet aspect physico-chimique de l'érosion est la théorie du processus en taux (Rate process theory) choisie par plusieurs auteurs (Ariathurai 1978; Arulanandan 1975; Gularte 1978 ; O'Neil 1979 ; Kelly et al. 1979 ; Clanton 1980). Cette théorie est basée sur le concept d'énergie d'activation $E$ et de volume d'écoulement $V_{f}$ (flow volume); $E$ est l'énergie nécessaire à fournir pour rompre les liens chimiques entre particules et agrégats de particules; $V_{f}$ est le volume unitaire libéré lorsque ces liens sont rompus.

Les auteurs cités ci-dessus relient alors la vitesse critique et le taux d'érosion à $E$ et $V_{f}$. Notons que ceux-ci interviennent directement sur la rhéologie des vases et que cette approche physico-chimique devrait pouvoir compléter l'étude proposée ici. Pour trouver une approche mécanique des processus d'érosion, il convient d'examiner quels types d'interaction peuvent avoir lieu entre l'eau et la couche de sédiments.
Supposons, en un premier temps, que l'érosion des sédiments cohésifs relève de l'arrachage de particules individuelles : il y aurait alors égalité entre la force tractrice du courant sur la particule : $F_{t}=\tau_{0} \times \Pi D^{2} / 4$ ( $D$ est le diamètre de la particule) et la force de résistance au mouvement des grains due au blocage par les autres grains : $F_{r}=f P=(1 / 6) \times \Pi \times f g\left(\rho_{s}-\rho_{e}\right) \times D^{3}$ ( $g$ est l'accélération de la pesanteur, $\rho_{s}$ et $\rho_{c}$ sont les masses volumiques du grain et de l'eau respectivement, $f$ est un facteur de proportionnalité); le seuil critique serait donc déterminé par l'équation

$$
\tau_{0}=(2 / 3) \times f g\left(\rho_{s}-\rho_{e}\right) D .
$$

La loi du début d'entrainement des matériaux pulvérulents relierait donc $D^{*}$, diamètre sédimentologique à $R^{*}$, nombre de Reynolds du grain dans la couche limite :

$$
D^{*}=D\left(\frac{\rho_{s}-\rho_{e} g}{\rho_{e} \nu^{2}}\right)^{1 / 3}
$$

$R * 2=2 f D^{2 * / 3}$ avec

$$
R^{*}=u_{*} D / \nu
$$

En fait, $f$ dépendrait de $R^{*}$ et il faut se reporter au diagramme de Shields (Fig. 6).

Soit une vase dont les grains ont un diamètre d'environ 10 microns ; $D^{*}$ vaut alors $10^{-8}$, ce qui donnerait $R^{*}$ de l'ordre de $10^{-11}$, c'est-à-dire une vitesse critique $V_{c}$ au plus de $10^{-9} \mathrm{~cm} / \mathrm{s} ! \ldots$

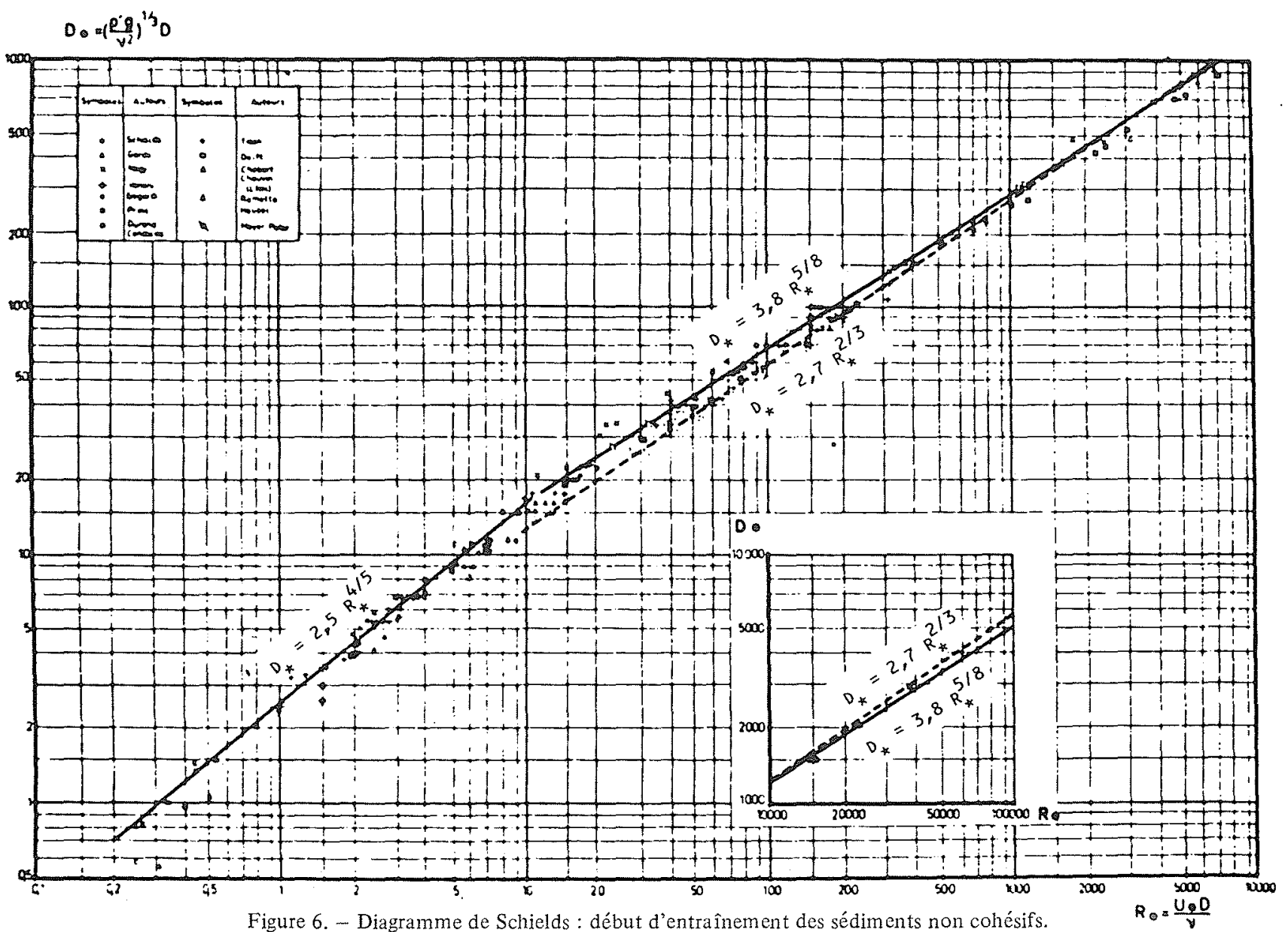


Le phénomène déterminant dans l'érodibilité de la vase est bien sûr la cohésion. Supposons maintenant que la vase ait un comportement de Bingham avec seuil plastique $\tau_{B}$. Plusieurs auteurs (Migniot 1968-1977; Gularte 1978; Clanton 1979; O'Neill, 1980) ont effectivement trouvé des relations expérimentales entre ce seuil et la vitesse critique d'entraînement.

Considérons le système décrit par la figure 7 . Trois cas peuvent se présenter suivant que la contrainte de cisaillement est supérieure au seuil nulle part (Fig. 7a), partiellement (Fig. 7b) ou partout (Fig. 7c). La vase ne commence à être entraînée qu'à la transition entre les phases $7 \mathrm{a}$ et $7 \mathrm{~b}$; le seuil critique serait alors:

$$
u_{* c}=\sqrt{\left\{h_{1} /\left(h_{1}+h_{2}\right)\right\} \tau_{B} / \rho_{1}}
$$
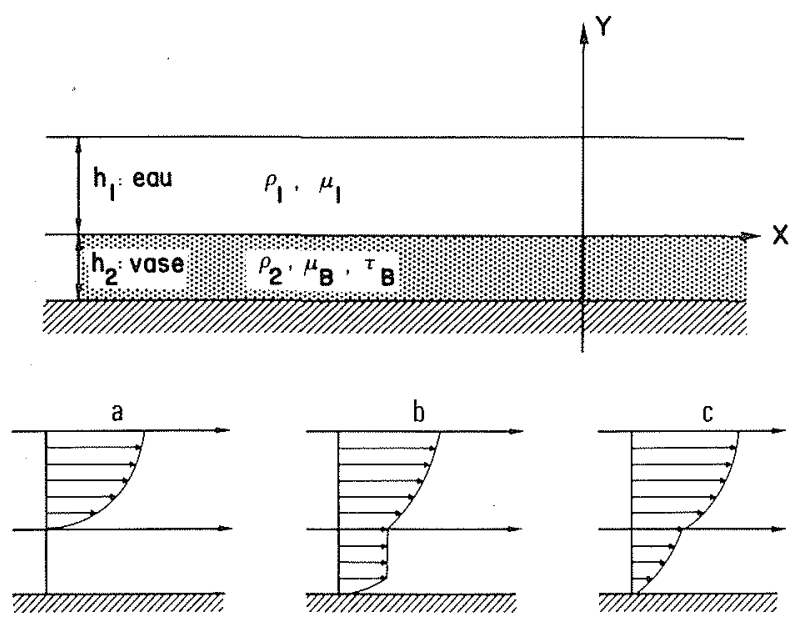

Figure 7. - Entraînement d'un fluide de Bingham par un courant.

Figure 7a: Pas d'entraînement

Figure $7 \mathrm{~b}:$ : Entrânement partiel

Figure $7 \mathrm{c}:$ En traînement total.

Dans Gularte (1978), les hauteurs $h_{1}$ et $h_{2}$ sont respectivement $10 \mathrm{~cm}$ et $5 \mathrm{~cm}$; on aurait alors $\tau_{C}=0,66 \tau_{B}$ ce qui donne deux ordres de grandeur de trop par rapport à ses résultats ex périmentaux.

Cet écart s'explique par le fait que le début d'entrấnement d'un fluide de Bingham met en jeu la cohésion de masse de l'ensemble de la vase alors que l'érosion est un phénomène plus ou moins superficiel.

\subsection{Description des mécanismes d'érosion}

Rôle des bouffées turbulentes : L'érosion est un processus intermittent (Sutherland, 1966) fortement relié à l'action des bouffées turbulentes (Nihoul, 1977; Mutlu Sumer et Beyhan Oguz, 1978). Dans le cas des sables, ces bouffées pourraient être responsables de la progres. sion des grains en saut de puce sur le fond, observable pour toute une gamme de vitesse du courant. Dans le cas des sédiments cohésifs, une particule arrachée sous l'action d'une bouffée turbulente reste en suspension dans le courant ; c'est pourquoi le seuil critique d'érosion est défini avec plus de précision pour les vases que pour les sables.

Par ailleurs, le rôle des bouffées turbulentes dans l'érosion des vases explique l'approche statistique de la détermination du taux d'érosion proposée par Partheniades (Einstein 1972) : étant donnée une distribution temporelle de la contrainte de cisaillement $\tau_{0}(t)$ sur le fonds et supposant connu le seuil critique d'érosion $\boldsymbol{T}_{\underline{e}}$ de la vase, le taux d'érosion est alors proportionnel à la moyenne de l'écart entre $\tau_{0}(t)$ et $\tau_{c}$ effectuée sur le temps pendant lequel cet écart est positif.

Deux processus d'érosion différents suivant la concentration en grains : Le deuxième point fondamental à retenir est la différence entre les processus d'érosion selon que les vases sont ou non concentrées.

Migniot (1968) a en effet observé cette différence; pour l'analyser, nous avons filmé l'érosion de vases préparées à différentes concentrations données dans le fond d'un canal. Nous présentons sur les figures 8 et 9 , deux séquences typiques d'expériences obtenues pour des vases de concentrations très différentes.

Dans le cas des vases faiblement concentrées (Fig. 8), on voit d'abord apparaittre une ondulation de l'interface; puis, celle-ci s'amplifie, déformant de plus en plus la couche de sédiments; ceux-ci sont finalement entraînés sous forme de filets de vase, puis dilués dans l'eau du courant.

Dans le cas des vases fortement concentrées (Fig. 9), des petits blocs de vase sont arrachés; une fois mis en suspension, ils restent individualisés dans l'eau du courant. Dans l'expérience présentée ici, ces blocs ont une taille de quelques millimètres, ce qui est bien suspérieur à la taille d'une particule (1 à 5 microns) ou même d'un agrégat de particules floculées (100 microns). Wan (1982) a par ailleurs observé l'érosion de vases concentrées par l'arrachage de blocs atteignant plusieurs centimètres.

Interprétation proposée : Ces observations nous ont suggéré l'approche suivante :

- les bouffées turbulentes sont considérées comme petite perturbation d'un écoulement uniforme horizontal permanent $V$ de l'eau ;

- dans le cas des vases peu concentrées, le critère d'éro. sion est ramené à un critère de stabilité ; au-delà d'une valeur critique $V$, les ondulations de l'interface s'amplifient dans le temps (Périgaud 1983, I) : $V$ est alors vitesse critique d'érosion ;

- dans le cas des vases concentrées, la petite perturbation induit des surpressions de l'eau interstitielle et des contraintes effectives qui déplacent l'eau et les grains séparément. Lorsque la vitesse $V$ est telle que la petite perturbation, d'échelle comparable à celle des bouffées turbulentes, se propage dans un tel milieu, les contraintes effectives vérifient le critère de Coulomb et il y a rupture de la vase sous forme de petits blocs (Périgaud 1983, II).

\section{Conclusion}

Ce papier se propose avant tout de faire le bilan des connaissances sur la rhéologie des sédiments cohésifs et leur processus d'érosion.

De multiples facteurs entrent en jeu à ce niveau, retenons cependant pour simplifier que sur le plan méca- 

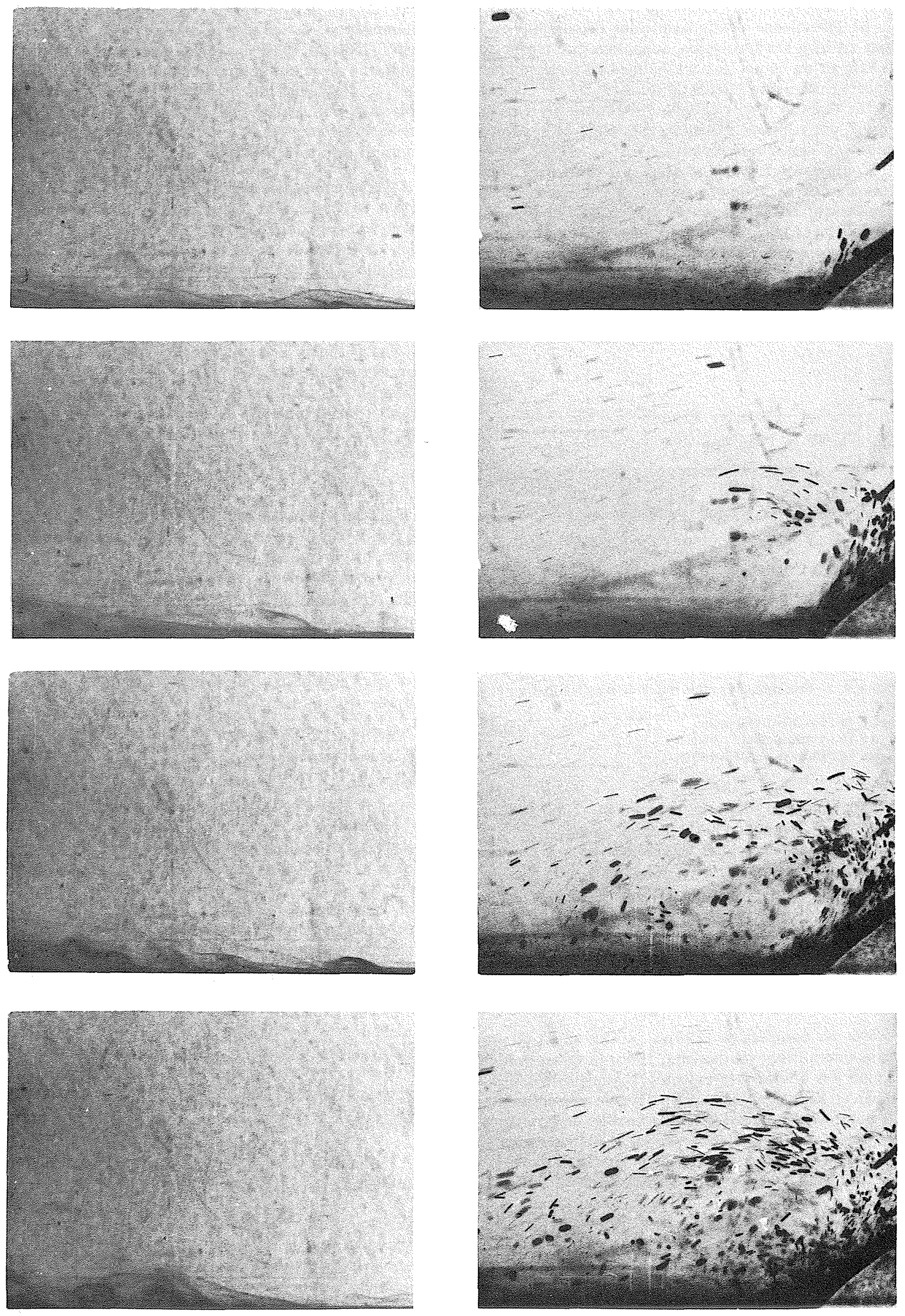

Figure 8. - Processus d'érosion pour des vases peu concentrées.

Figure 9. - Processus d'érosion pour des vases très concentrées. 
nique, les vases ont un comportement et une érodibilité bien différents selon qu'elles sont ou non concentrées en sédiments secs.

Les vases peu concentrées ont un comportement de type fluide visqueux; on ne peut cependant pas parler de viscosité newtonienne : le comportement d'une vase n'est pas linéaire (loi puissance ou modèle de NortonHoff); parfois même, il montre un seuil plastique (modèle de Bingham). L'érosion de ces vases apparaît audelà d'une vitesse critique, de manière intermittente : l'interface eau-sédiment est déformée sous forme d'ondulations qui s'amplifient et se tordent en filets de vase déchirés par le courant.

Les vases concentrées ont un comportement complexe moins bien connu; l'étude au scissomètre montre que les contraintes effectives s'exerçant sur les grains ne peuvent dépasser un seuil qui est la cohésion. La rupture a lieu sous forme de blocs à l'endroit où les contraintes effectives vérifient le critère de Coulomb. L'érosion se fait en effet au-delà d'une certaine vitesse critique, de façon intermittente et sous forme de blocs de vase arrachés par le courant.

Le caractère intermittent des processus d'érosion peut être relié à l'action des bouffées turbulentes qui s'exercent sur l'interface eau-sédiment, comme sur toute paroi développant une couche limite dans un écoulement turbulent. Ces bouffées turbulentes font l'objet de nombreuses recherches experimentales et théoriques. On comprend pourquoi la complexité des actions hydrodynamiques turbulentes aussi bien que celle de la rhéologie des sédiments cohésifs rend toute approche théorique des processus d'érosion encore très incertaine.

\section{Remerciements}

Je tiens à remercier le Professeur J.-S. Darrozes qui a dirigé ce travail ainsi que le Docteur C. Migniot qui m'a conseillé dans la partie expérimentale de ce travail.

Je voudrais également souligner l'aide apportée par le laboratoire de Physique et Chimie Marines et l'Ecole Nationale des Ponts et Chaussées. Je remercie enfin le Docteur J.-F. Minster pour avoir amélioré le manuscrit.

\section{Références bibliographiques}

ARIATHURAI R., ARULANANDAN K. - 1978, Erosion rates of cohesive soils. J. Hyd. Div., Vol. 104-HY2, p. 279-283.

ARULANANDAN K., LOGANATHAN P., KRONE R.B. 1975 , Pore and eroding fluid influences on surface erosion of soils. Journal of Geotechnical engineering division ASCE. Vol 101, $n^{\circ}$ GT1, p. 51-66.

BIAREZ J. - 1977, Mécanique des sols en mer profonde. Etude réalisée sous la direction du professeur J. Biarez. Contrat CNEXO, (pp. 208)

CANTWELL B. - 1981, Organized motion in turbulent flow. Ann. Rev. Fluid. Mech., Vol 13, p. 457-515.

CHAVARRIA F.E. - 1980, Etude rhéologique des suspensions de flocs biologiques. Thèse de Docteur Ingénieur à l'Université de Montpellier, (pp. 95).

CHONG J.S., CHRISTIANSEN E.B., BAER A.D. - 1971, Rheology of concentrated suspensions. Journal of applied polymer science vol 13, p. 2007-2021.

CLANTON M.L. - 1980, Application of rate process theory to erosional parameters of a deep sea pacific sediment. PhD thesis. University of Rhode Island, (pp. 148).
DARROZES J.S., FRANCOIS C. - 1982, Lecture notes in physics. Vol 163, Mécanique des fluides incompressibles. Springer Verlag. Berlin Heidelberg. New York.

DOLIGASKI T.L., SMITH C.R., WALKER J.D.A. - 1979, Production mechanism for turbulent boundary layer flows. Symposium on viscous drag reduction. Dallas, Texas, Vol. 72.

DUNN A. -1959 , Tractive resistance of cohesive channels. Journal of the Soil Mechanics and Foundations. Division ASCE, Proc. 85 (SM3).

EINSTEIN H.A. - 1972, Sedimentation symposium, Colorado State University.

GERARD B. - 1978, Les nodules polymétalliques de la zone Clarion Clipperton. Aspects geotechniques des boues argileuses. Rapport CNEXO-COB.

GILBERT F. - 1983, Definition of mechanical quantities on macroscopic scale for multiphase media. Submitted to C.R. Acad. Sc. Paris, Série 2.

GULARTE R.C. - 1978, Erosion of cohesive marine sediments as a rate process. PhD in ocean engineering. University of Rhode Island, (pp. 120).

HOFF N.J. - 1954, Approximate analysis of structures in the presence of moderately large creep deformations, Quat. Appl. Math., Vol. 12, n 1, p. 49.

JEFFREY D.J., ACRIVOS A. - 1976, The rheological properties of suspensions of rigid particles. A.I.Ch.E. Journal, Vol. $22, \mathrm{n}^{\circ} 3$

KELLY W.E., GULARTE R.C., NACCI V.A. - 1979, Erosion of cohesive sediments as a rate process. $J$, Geotch. Engineering Division, Vol. 105, CT5 p. 673-676.

KLINE S.J., REYNOLDS W.C., SCHRAUB F.A., RUSTADLER P.W. - 1967, The structure of turbulent boundary layers. Journal of Fluid Mechanics. Vol. 50 part 1, p. 133-160.

KRONE R.B. - 1962, Flume studies of the transport of sediment in esturial shoaling processes. Final Report Hydr. Eng. and Sanitary Eng. Res. Lab. University of California, Berkeley.

LAMBERMONT J., LEBON G. - 1978, Erosion of cohesive soils. Journal de Recherches Hydrauliques, Vol. $16 \mathrm{n}^{\circ} 1$, p. 27-44.

MADSEN O.S. - 1978, Wave induced pore pressures and effective stresses in a porous bed. Geotechnique, Vol. $28, n^{\circ} 4$, p. $377-393$.

McCAVE I.N. - 1978, Sediments in the abyssal boundary layer. Oceanus, Vol. $21 \mathrm{n}^{\circ} 1$, p. 27-33.

MCCAVE I.N., SWIFT S.A. - 1976, A physical model for the rate of deposition of fine grained sediments in the deep sea. Geological Society of American Bulletin, Vol. 87, p. 541. 546.

MEHTA A.J., PARTHENIADES E. - 1979, Kaolinite resuspension properties. Jour. of the Hydraulics Division HY 4 vol 105, p. 411-416.

MEI C.C., FODA A.M. - 1980, Boundary layer theory of waves in a poroelastic sea bed. To appear in: Soil behaviour under transcient of cycling loading. Edition Zienkiewics/Pande Wiley In tersience.

MIGNIOT C. - 1968, Etude des propriétés physiques de différents sédiments très fins. La Houille Blanche. Vol. 1., p. 591 620.

MIGNIOT C. - 1970, Etude des propriétés physiques de la vase de la Gironde. Tassement et rigidité initiale. Commande EDF HC 17230 , pp. 44.

MIGNIOT C. - 1977, Action des courants, de la houle et du vent sur les sédiments. La Houille Blanche $\mathrm{n}^{\circ} 1$, p. 9-47.

MITCHELL J.K. - 1977, Fundamentals of soil behaviour John Wiley N-Y.

MONIN A.S., YAGLOM A.M. - 1971, Statistical fluid mechanic. The MIT Press.

MOORE W.L., MASCH F.O. - 1962, Experiments on the scour resistance of cohesive materials. Journal of Geophysical Research Vol. 67, n 4, p. 1437-1499.

MUTLU SUMER B., BEYHAN OGUZ. - 1978, Particle motions near the bottom in turbulent flow in an open channel. Jour. nal of Fluid Mechanic, Vol. 86, part. 1, p. 109-127.

NIHOUL J.C. - 1977, Turbulent boundary layer bearing silt in suspension. The Physics of Fluids, Vol. 20, $\mathrm{n}^{\circ} 10$ Part. II. 
OLDROYD J.G. - 1947, A rational formulation of the equations of plastic flow for a Bingham solid. Proc. Cambridge Philosophy Society, Vol. 43

O'NEIL G.M. - 1979, Interactions of $\mathrm{pH}$, salinity and mineralogy upon the rheological properties of clay suspension. PhD University of Rhode Island, pp. 85 .

PARCHURE M. - 1980, Effect of bed shear stress on the erosional characteristics of kaolinite. $\mathrm{PhD}$ thesis. University of Florida.

PARSONS J. - 1981, La mobilité de la vase. La technique de l'eau et de l'assainissement, $n^{\circ} 414 / 415, p .43-47$.

PARTHENIADES E. - 1965, Erosion and deposition of cohesive soils. Journal of the Hyd. Div. Proceeding of the ASCE. HY 14204 , p. 105-137.

PERIGAUD C. - 1984, Erosion of cohesive sediments by a turbulent flow, Part. 2 : High concentration. Journal de Mécanique théorique et Appliquée, $n^{\circ} 4$.

PERIGAUD C. - 1984, Erosion of cohesive sediments by a turbulent flow. Part. 2: High concentration. Journal de Mécanique théorique et Appliquée, $n^{\circ} 4$.

RAUDKIVI A.J., HUTCHINSON D.L. - 1974, Erosion of kaolinite by flowing water. Proc. Royal Society A 337, p. 537 . 557.

RICHARDS A., PARKS J. - 1976, Marine geotechnology. The Benthic boundary layer. Edité par McCAVE, Plenum Press, NY et Londres, p. 157-181.

SCHLICHTING H. - 1979, Boundary layer theory. McGraw Hill. Series in Mechanical Engineering.

SCHLOSSER F, - 1978, Cours sommaire de mécanique des sols. Ecole Nationale des Ponts et Chaussées.

SCHOWALTER W.R. - 1978. Mechanics of non newtonien fluids. Pergamon Press. pp. 300.
SILVA A., HOLLISTER C. - 1979, Geotechnical properties of ocean sediments recovered with the giant piston corer: Blake Bahama outer ridge. Marine Geology, p. 1-22.

SILVA A., HOLLISTER C., LAINE E. et BEVERLY B. - 1976, Geotechnical properties of deep sea sediments: Bermuda Rise. Marine Geotechnology, Vol. 1, p. 195-232.

SOUTHARD J.B. - 1973, Erodibility of fine abyssal sediments. Deep sea sediments, Plenum Publishing Corporation N.Y. Edited by A.L. Inderbitzen, p. 367-379.

SUTHERLAND A. - 1966, Entrainement of fine sediments by turbulent flows. PhD thesis M.I.T.

TERZAGHI K. - 1960, From theory to practice in Soil Mechanics, John Wiley and Sons. New-York.

THOMAS D.G. - 1961, Laminar flow properties of floculated suspensions. A.I.C.H.E. Journ., Vol. 17, p. $431-437$.

THOMAS D.G. - 1963, Transport characteristics of suspension. Relation of hindered settling floc characteristics to theological parameters, A.I.C.H.E. Journ. Vol. 9, p. 310-316.

VALEMBOIS J., MIGNIOT C. - 1975, Rejets des produits de dragage à l'aval d'un barrage sur l'oued Hamiz. La Houille Blanche, Vol. $2 / 3$, p. 105-172.

WILLMARTH W.W. - 1975, Pressure fluctuations beneath turbulent boundary layers. Ann. Rev. of Fluid Mechanic, Vol. 7 , p. 13-37.

YAMAMOTO T. - 1977, Wave induced instability in seabeds. Symposium on Coastal Sediments, Charleston, p. 898-913.

YOUNG R.N. et SOUTHARD J.B. - 1978, Erosion of fine grained marine sediments. Sea floor and laboratory experiments. Geological Society of American Bulletin, Vol. 89, p. 663-672.

WAN Z. - 1982, Bed material movement in hyperconcentrated flow. Institute of hydrodynamics and hydraulic Engineering. Technical University of Denmark. Lyngby, pp. 79. 